\title{
L'enseignement de la morale au quotidien : le rôle des inspecteurs primaires, 1880-1914
}

Primary school inspectors and the teaching of morals under the French Third Republic

Grundschulinspektoren und Moralunterricht unter der 3. Republik

Los inspectores de la enseñanza primaria y la enseñanza de la moral bajo la

Tercera República

\section{Delphine Mercier}

\section{OpenEdition}

\section{Journals}

\section{Édition électronique}

URL : https://journals.openedition.org/histoire-education/1308

DOI : 10.4000/histoire-education. 1308

ISSN : 2102-5452

\section{Éditeur}

ENS Éditions

\section{Édition imprimée}

Date de publication : 1 janvier 2005

Pagination : 45-66

ISBN : 2-7342-1006-1

ISSN : 0221-6280

\section{Référence électronique}

Delphine Mercier, «L'enseignement de la morale au quotidien : le rôle des inspecteurs primaires,

1880-1914 ». Histoire de l'éducation [En ligne], 105 | 2005, mis en ligne le 31 décembre 2009, consulté le 20 mai 2021. URL : http://journals.openedition.org/histoire-education/1308 ; DOI : https://doi.org/ 10.4000/histoire-education.1308 


\section{L'ENSEIGNEMENT DE LA MORALE AU QUOTIDIEN : le rôle des inspecteurs primaires (1880-1914)}

par Delphine MERCIER

L'enseignement de la morale, tel qu'il est institué à partir de 1882 par le régime républicain, constitue pour les historiens l'occasion de s'interroger sur le triomphe de l'idée laïque dans l'école de la Troisième République (1). Privilégier les discours ministériels et les textes officiels tend cependant à réduire l'œuvre éducative de la Troisième République à sa politique législative. L'étude des manuels scolaires ou des articles de périodiques professionnels (2) néglige quant à elle les adaptations des attentes gouvernementales «au monde de la classe», soit à cette «réalité empirique vécue à l'école» (3).

L'enseignement de la morale est autant une éducation qu'une instruction. Placé en tête des programmes de 1882, il coordonne toute la formation primaire puisqu'il vise à transcrire, dans les actes quotidiens de chacun, les connaissances qu'apportent les différents apprentissages scolaires. Objet d'un enseignement propre, la morale n'est pas seulement une instruction, subordonnée à des leçons données à heure précise, voire à la mémorisation de quelques maximes. À l'œuvre dans toutes les matières (4), elle utilise, complète, relie les connaissances et les aptitudes particulières que développent les autres enseignements.

(1) Les ouvrages de J.-M. Mayeur: La question lä̈que - XIXe-XXe siècles, Paris, Fayard, 1997, de J. Baubérot, G. Gauthier, L. Legrand et P. Ognier: Histoire de la laïcité, Besançon, CRDP, 1994 et de Y. Déloye : École et citoyenneté, Paris, FNSP, 1995 permettent d'établir les fondements et les finalités éducatives des Républicains.

(2) Nous ne pensons pas que «pour savoir [...] comment s'enseigne la morale [...] il [suffise] d'ouvrir un manuel de morale», ainsi que l'affirme L. Maury: L'enseignement de la morale, Paris, PUF, 1999.

(3) J. Baubérot: La morale lä̈que contre l'ordre moral, Paris, Seuil, 1997, pp. 20-22.

(4) Les finalités et les contenus de l'histoire et de l'instruction civique visent ainsi à inculquer cet idéal moral, en constituant, par le biais de leurs acteurs ou de leurs institutions, un corpus exemplaire de vertus. 
Envisager cet enseignement en termes de pratiques scolaires concrètes nécessite d'adjoindre aux sources traditionnellement utilisées, l'étude de documents qui permettent une approche plus fine de ce qui se passait dans les classes. Cette démarche, adoptée par certains historiens dans leurs travaux les plus récents (1), nécessite que le corpus utilisé soit suffisamment riche, quantitativement et qualitativement, pour offrir toutes les chances de représentativité et de pertinence. Les documents laissés par l'inspection primaire ont ces deux mérites. Croisés avec d'autres sources, ils permettent d'appréhender les réalités des pratiques de classe et la réflexion mise en œuvre pour les améliorer (2).

\section{L'INSPECTION PRIMAIRE ET L'ENSEIGNEMENT DE LA MORALE}

Deux raisons mènent à privilégier les sources laissées par l'inspection primaire. D'une part, le statut même de leurs auteurs dont la fonction est, dès sa création, liée à la politique éducative gouvernementale. D'autre part, la diversité (3) et la qualité de leur conservation, que l'on observe aussi bien à l'échelon départemental que national (4).

(1) B. Dancel (pour l'enseignement de l'histoire) comme J. Baubérot (pour l'enseignement de la morale) ont ainsi utilisé, respectivement, des copies du certificat d'études et des cahiers d'élèves. Leur intérêt supérieur réside dans ce qu'ils livrent de la compréhension et de la mémorisation des leçons du maître ou du manuel par les élèves. Davantage révélateurs des savoirs retenus par certains que des savoirs enseignés à tous, ces documents ne confrontent pas l'historien à la diversité des pratiques de classe, aux méthodes pédagogiques employées.

(2) Pour une présentation méthodologique précise de l'ensemble de ces sources: D. Mercier: L'inspection primaire, l'enseignement de l'histoire et de l'instruction morale et civique (1880-1914), thèse de doctorat, Paris IV Sorbonne, décembre 2002.

(3) Pour le département de la Seine-et-Oise: rapports d'inspection (référencés: R.I., nom de l'inspecteur primaire, date), rapports annuels de circonscriptions (référencés: R.A., nom de l'inspecteur primaire, date) et conférences pédagogiques de la circonscription de Pontoise.

(4) Le travail repose ici sur un dépouillement complet des documents de l'inspection primaire du département de la Seine-et-Oise (T SUP 130-132 et T DI 1 à 83). Pour plus de pertinence, cette analyse est mise en regard avec des sources nationales (F 17 11630 - rapports généraux sur l'enseignement de la morale dans la Seine de 1900 à 1912 et 71 AJ 19 - enquête nationale sur l'enseignement de la morale de mars 1889). Les documents de l'inspection générale et académique de la Seine-et-Oise et de la Seine (référencés: R.I.A. ou R.I.G., département, date) ont été pris en compte, dans la mesure où ils s'appuient sur les documents fournis par l'inspection primaire, qu'ils citent d'ailleurs très souvent. 
Les choix éducatifs engagés dans la décennie 1880 ont renforcé le rôle dévolu aux inspecteurs depuis 1835. Le doublement de leur nombre (1), la multiplication de leurs tâches et l'assurance de leur compétence éprouvée par un examen rigoureux, témoignent du souhait des Républicains de faire du travail quotidien de ces fonctionnaires un outil au service de la réussite des réformes de l'école primaire. À partir de cette date, l'inspection a pour principale mission d'ancrer les nouveaux objectifs de formation dans les pratiques enseignantes; elle voit, par conséquent, sa fonction pédagogique développée. Ainsi, dans les rapports d'inspection, l'espace réservé à l'observation des séquences et aux conseils de pédagogie pratique prédomine sur les questions administratives, l'examen physique et le jugement de la moralité de l'enseignant. Une semblable évolution des préoccupations professionnelles se retrouve dans les conférences pédagogiques. Rétablies en 1880 , ces réunions offrent à l'inspecteur la possibilité d'unifier l'enseignement donné puisqu'elles réunissent tous les maîtres d'une circonscription. À partir de 1892, par exemple, les questions soumises par l'inspecteur de la circonscription de Pontoise aux instituteurs qu'il réunit en conférence vont au-delà d'un simple commentaire des textes réglementaires sur l'enseignement moral ou de l'élaboration d'un dogme qui limiterait les initiatives enseignantes. Les conférences pédagogiques de ce département (2) comportent des discussions théoriques qui invitent les enseignants à réfléchir aux objectifs de la formation morale, les incitent à comparer leurs procédés, leurs outils pédagogiques, c'est-à-dire à juger de l'efficacité de leur méthode.

Le développement de la fonction pédagogique permet à l'inspection primaire d'intervenir dans un domaine qu'elle connaît bien car elle l'a expérimenté (3) et y a réfléchi: la pratique de l'enseignement au quotidien. Conduits à s'interroger sur les résultats de l'enseignement, les inspecteurs orientent volontiers leur discours sur la façon d'enseigner. Qu'il s'agisse des bilans adressés annuellement à

(1) On constate une augmentation de $128 \%$ entre 1842 et 1914 . En 1878 , le nombre d'inspecteurs en poste est de 385 contre 469 en 1886.

(2) Ces conférences dépassent la seule critique, par un supérieur hiérarchique, de la leçon exposée par un maître devant un auditoire de collègues (procédé le plus communément utilisé dans les conférences de l'époque). On note l'exceptionnelle qualité des échanges entre enseignants, inspecteur primaire et inspecteur d'académie lors des conférences pédagogiques de la circonscription de Pontoise.

(3) $70 \%$ des inspecteurs du département de la Seine-et-Oise, entre 1880 et 1914 , sont d'anciens instituteurs. 
l'administration supérieure, de leurs collaborations aux revues pédagogiques ou de la rédaction de manuels scolaires (1), ils témoignent de leur souci de faire évoluer les méthodes pédagogiques.

L'inspection primaire a pour mission de faire comprendre à tous les acteurs du système éducatif primaire - enseignants, élèves, familles et élus - les idéaux de la formation morale voulue par les législateurs. Dans l'enseignement moral plus que dans toute autre discipline, ce fonctionnaire devient l'agent des ambitions éducatives de la République, quand il n'incarne pas l'image de la morale républicaine. Par une communauté de vue avec le monde des «primaires», par une connaissance des populations locales et une compréhension des besoins de l'enseignement au quotidien, l'inspection peut, par une «attitude réservée» (2) et en mesurant ses propos, agir efficacement. Dans cette mission, les inspecteurs témoignent de la nécessité d'adapter les objectifs de la nouvelle éducation morale à la pluralité des situations scolaires.

\section{LES PRIORITÉS DE L'INSPECTION}

Il ne s'agit pas ici d'exposer la pluralité des doctrines qui soustendent l'enseignement de la morale laïque - les enseignants n'ayant d'ailleurs pas à en «discuter les bases philosophiques» (3) dans leurs classes - mais il est important de souligner en quoi les nouveaux objectifs de formation constituent une gageure éducative qui donne aux inspecteurs un rôle important. Les programmes de 1882, comme la lettre de Jules Ferry aux instituteurs de 1883, insistent avant tout sur l'idée d'enseigner une morale consensuelle. Dans les principes qu'elle emprunte au Décalogue, la morale laïque propose, en effet, des «règles élémentaires de la vie morale », c'est-à-dire des préceptes à valeur universelle, acceptables par tous, du moins par tout «honnête

(1) Moyen pour les inspecteurs d'obtenir un complément à son traitement de fonctionnaire, la rédaction d'ouvrages scolaires implique cependant un surcroît de travail que ne justifie pas la diffusion relativement faible de leurs manuels, du moins comparativement à ceux des grands noms de la pédagogie. Écrire des livres scolaires ou des ouvrages de didactique consiste, pour eux, à traduire dans leurs écrits la part de leur expérience et de leur réflexion pour venir en aide aux maîtres comme aux élèves.

(2) G. Rossignol: «Notes et conseils d'inspection», BDIP de l'Indre, mars 1912, p. 58: l'inspecteur évitera de «donner un conseil que tel de ses prédécesseurs a condamné d'avance ou que son successeur jugera détestable».

(3) Lettre de J. Ferry aux instituteurs, 1883. 
homme» (1). Insister sur les devoirs qui rapprochent les hommes, et non sur les dogmes qui les divisent, revient, pour les maitres, à faire de cet enseignement un outil pour corriger les défauts des enfants et développer leurs qualités (2). Présentée comme la «bonne et antique morale de nos pères », on peut alors s'interroger sur les débats qu'elle a suscités entre ses partisans et ses détracteurs. Si elle n'enseignait rien de neuf, comment a-t-elle pu laisser les enseignants perplexes, voire démunis? Morale spiritualiste, acceptée par Jules Ferry dans un souci d'apaisement, cet enseignement se distingue cependant de celui qui l'a précédé par son indépendance des croyances religieuses et par ses fondements empruntant aussi bien à la philosophie des Lumières, qu'aux idéaux de la Révolution, au kantisme ou au positivisme. Construite sur la suprématie de la raison, le respect de la dignité humaine et la primauté des libertés individuelles, cette morale «sans épithète» ne répond pas seulement au souhait d'une normation des comportements individuels, garante de la conservation de l'État. Elle possède une finalité supérieure dont les «premières vérités » sont les «notions du devoir et du droit» (3). Plus qu'une simple instruction ou un nouveau catéchisme, ce nouvel enseignement établit les obligations morales des enfants dans la famille et dans l'école et fonde, à terme, pour les adultes, une succession de devoirs individuels et sociaux. D'ailleurs, les pédagogues ne s'y trompent guère, qui savent qu'instruire sur l'histoire du progrès national, sur l'œuvre de la République et sa conquête des libertés, voire sur les idées d'une morale revue par la laïcité, reste insuffisant. Pour l'inspecteur Boyer, l'enseignement moral «n'est pas seulement la connaissance du devoir qu'il faut donner, mais c'est l'amour, l'habitude du bien qu'il faut inculquer» (4). Faire adhérer le futur citoyen relève plus de l'ordre du «faire vouloir» que du «faire savoir», situation qui nécessite une adhésion pleine et entière du maître et une pratique de l'élève. Or, sur ce point particulier, c'est bien à l'inspection primaire que l'on confie le rôle de modifier les habitudes d'enseignement pour les mettre en conformité avec les finalités éducatives visées. De plus, les arrêtés ministériels de 1882 et 1887 qui fixent l'organisation pédagogique et les programmes de l'enseignement de la morale, restent très flous car

(1) Ibid.

(2) Le succès du manuel de l'inspecteur primaire Cuir, intitulé Les Petits écoliers - lectures morales sur les défauts et les qualités des enfants, souligne que cet aspect de l'enseignement moral est le mieux compris et le mieux accepté. Publié pour la première fois en 1874, cet ouvrage est constamment réédité jusqu'en 1904.

(3) Lettre de J. Ferry aux instituteurs, 1883.

(4) Boyer: Le livre de morale des écoles primaires, 1895. 
ils mêlent objectifs, contenus et méthodes d'enseignement. Distinguer, dans l'acte quotidien d'enseignement, les finalités éducatives des contenus à enseigner et des procédés pédagogiques à suivre n'est pas une tâche facile pour les maîtres; c'est, en revanche, l'une des missions confiées à l'inspection primaire.

Dans les contenus d'enseignement propres à la morale laïque, les inspecteurs ne se distinguent pas véritablement des autres pédagogues (1). Deux choix sont cependant suffisamment marqués et communément partagés par ce corps de fonctionnaires. D'une part, les inspecteurs primaires n'ont de cesse de rappeler que cet enseignement doit être bâti sur le «principe fondamental» de «l'excellence de la raison» et sur ceux de la «dignité humaine» et de la «solidarité sociale» (2). D'autre part, ils témoignent d'un souci constant de mettre ces principes à la portée des élèves, sans excès de simplification et en respectant leur conscience (3). C'est donc le refus du dogmatisme et le respect de l'enfant qui sont privilégiés.

Ces deux axes apparaissent clairement dans les thèmes du patriotisme et de la charité (4). D'abord développé comme un lien affectif comparable au lien familial, le patriotisme que les inspecteurs proposent, dès 1895, a des fondements plus institutionnels (5). Les inspecteurs affirment la primauté de la communauté politique et civique sur le lien des générations et l'héritage des ancêtres (6). Leurs références à l'œuvre de la Révolution française, aux libertés fondamentales qui en résultent et qui s'incarnent dans les institutions de la Troisième

(1) Du moins par rapport aux contenus historiques où l'inspection primaire témoigne très tôt, et de manière plus marquée, de sa spécificité.

(2) R.I., Lottin, 1907-1908 et 1909.

(3) Les inspecteurs déplorent que la leçon de morale «tombe dans les côtés mesquins et puérils» (R.I., Boë, 1890) que l'élève vienne à considérer la morale «comme une théorie qu'il ne convient de pratiquer que sous bénéfice d'inventaire» (R.I.A., Seine, 1912). Ils sont satisfaits lorsque les maîtres respectent "la personnalité de l'enfant, sa dignité d'homme en formation » (R.A., Plâtrier, 1892).

(4) L'analyse de ces thèmes repose sur une étude comparative de 33 manuels d'inspecteurs et de 10 manuels de référence les plus répandus dans les écoles publiques car publiés par des pédagogues de renom.

(5) La communauté politique, institutionnelle et civique est ce qui fonde le patriotisme dans près de $80 \%$ des manuels d'inspecteurs, contre à peine $30 \%$ dans les manuels de référence.

(6) Sur le rôle du culte des héros dans la formation morale républicaine, cf. J.-F. Chanet: «La fabrique des héros - Pédagogie républicaine et culte des grands hommes de Sedan à Vichy », Vingtième siècle, n ${ }^{\circ} 65$, janvier-mars 2000. 
République sont une constante. On passe de la notion de patrie guerrière à celle de patrie du progrès: pour les inspecteurs, c'est l'histoire institutionnelle de la France qui justifie qu'elle devienne le symbole d'une patrie libre. Après 1910, l'inspection développe systématiquement dans ses manuels l'idée que le patriotisme repose avant tout sur des liens sociaux et civiques librement consentis. Solidarité d'intérêts et d'espoirs, la patrie se confond alors avec un État républicain dont les bienfaits sont source de l'union nationale. Faisant la part du chauvinisme et de l'internationalisme, les inspecteurs exposent, avec davantage de régularité et de force que les universitaires, le recours à l'arbitrage international et à l'idée d'une fraternité universelle comme premiers principes pour éviter les guerres.

Cette conception modérée qu'ont les inspecteurs du patriotisme montre qu'ils sont en accord avec une grande majorité des enseignants. Reste alors à trouver les moyens d'adapter ce discours aux capacités des élèves auxquels il s'adresse. Privilégier une position raisonnée comme fondement à cette obligation de conscience conduit les inspecteurs à développer plus spécifiquement certains actes patriotiques attendus des enfants. Objectif fondamental de la formation morale primaire, le rapport au travail, scolaire puis professionnel, forge ainsi ce lien social qui justifie le patriotisme. L'adaptation à la vie de l'école et l'uniformisation des comportements qu'elle implique constituent, pour tous les pédagogues, un prélude à l'intégration de l'enfant dans la société. Redéfinir les attitudes quotidiennes de l'élève revient à former alors des écoliers modèles, dans l'espoir d'en faire, plus tard, des citoyens libres et utiles car instruits et éclairés. L'obéissance restant la principale vertu scolaire (1), les attitudes requises de l'élève sont donc souvent énoncées sous la forme de préceptes auxquels l'enfant doit se conformer, de manière mécanique et docile. Adoptant également cette forme de leçon, les inspecteurs insistent néanmoins sur les raisons de réussir cette étape. Pour eux, l'école de la République «remplit (là) avec générosité son devoir social» et c'est pour cela qu'une reconnaissance particulière lui est due. Lieu «d'apprentissage de la vie sociale» (2), l'école tisse la première ce lien entre droit et devoir. Le thème du progrès national se relit alors

(1) Parmi les devoirs envers le maître, l'obéissance est la vertu le plus souvent mentionnée dans l'ensemble des manuels $(29,8 \%)$ suivie de la reconnaissance $(24,8 \%)$ et du respect $(23,2 \%)$.

(2) Maxime de morale - cahier de roulement CM1, 1909-1910, école de Pontoise. 
autrement: constitué par le droit de chacun à s'instruire et l'offre faite à tous d'une progression sociale, il impose, réciproquement, des devoirs.

Le thème de la charité est, par excellence, celui où le nouvel enseignement moral doit faire preuve de sa spécificité. Son traitement, dans l'ensemble des manuels, souligne la réappropriation, par l'école laïque, des anciennes valeurs morales. Abordée après les chapitres sur les «devoirs individuels» qui doivent perfectionner l'âme et le corps, la charité prend place dans le cadre de l'étude des «devoirs sociaux». Vertu chrétienne par excellence (1), elle est reformulée par les pédagogues républicains comme cette «sœur de la justice et mère de la fraternité » (2). «Impératif catégorique » supérieur à la loi parce que volontaire et libre, cette obligation morale ne peut être le fait que d'individus conscients de leurs devoirs, indépendamment d'autorités divines ou temporelles. En raison même de son caractère facultatif, car non soumis aux sanctions de la loi, l'exercice de cette vertu reste difficile. Jugée comme un acte n'allant pas de soi, la charité mérite par conséquent d'être expliquée (3) par une suite de comportements pratiques (4). Dans leurs manuels, les inspecteurs suivent cette procédure, mais ils cherchent également à lutter contre une simplification abusive de l'exercice de la charité par des leçons raisonnées. Leur objectif est d'habituer l'esprit enfantin à des réflexions sur cette pratique plus élevées que celles suscitées communément par les historiettes-modèles utilisées. Redéfinir cet acte consiste pour ces auteurs à associer, pour mieux les distinguer, les devoirs de charité et de justice. C'est là le point d'orgue de l'éducation morale telle qu'ils la conçoivent: si la justice fait respecter l'égalité établie par la loi, la charité tend à compenser, par des dévouements personnels, les inégalités sociales et poursuit une œuvre de fraternité.

(1) Elle puise ses origines dans un héritage chrétien que tous reconnaissent car il concourt à la cohésion voulue par la morale laïque. Cependant, les auteurs, quelle que soit leur origine, ne font que très rarement référence au Décalogue ou aux Évangiles.

(2) Poignet et Bernat: Le livre unique de morale et d'instruction civique, 1905.

(3) Différentes représentations exemplaires de la charité sont proposées: les actes de dévouement, de sacrifice, de bienfaisance, de clémence, d'assistance - physique ou matérielle -, de compassion, d'indulgence, de pardon sont donnés aux enfants comme supports à son explication.

(4) Parmi ces derniers se distingue l'aumône dont la reformulation devient un symbole de la relecture laïque de cette vertu. 
Mettre en place les devoirs qu'exige la formation morale, c'est-àdire faire pratiquer ces vertus, est donc un souci qui appartient en propre aux pédagogues les plus engagés dans l'école. On comprend mieux dès lors que l'inspection primaire se soit particulièrement attachée à une réforme des méthodes d'enseignement propres à cette discipline.

\section{RÉFORMER LES MÉTHODES D'ENSEIGNEMENT}

Les remarques des inspecteurs sur les pratiques des instituteurs font apparaître trois défauts: tout d'abord, un excès de simplification qui ne tend pas à une «moralité très élevée» (1); ensuite, un dogmatisme sentencieux qui conduit trop souvent à des cours de morale théorique; enfin, une monotonie de ton comme de propos qui traduit souvent l'indifférence du maître pour cet enseignement. Pour éviter ces erreurs, les inspecteurs rappellent qu'il faut faire, deux fois par semaine, de véritables leçons dont le plan idéal devrait se composer de quatre parties. Un exemple au départ qui «précise les idées» ou «propose un problème moral»; un entretien, ensuite qui conduit l'élève à prendre une part active en «apportant sa réflexion» et en retrouvant «sans peine les arguments développés par le maître»; puis, des interrogations pour conduire «les enfants à rappeler des exemples analogues, des souvenirs de lectures ou d'exercices écrits»; enfin, un résumé écrit qui «laisse une trace tangible» (2). Le cours peut, en outre, se conclure par une lecture, «choisie le plus souvent pour émouvoir» (3) ou bien par des récits narrés.

À la fois instruction et éducation, cet enseignement nécessite de tout pédagogue qu'il puisse, en théorie, distinguer l'une de l'autre. Il ne doit cependant pas «les séparer dans la pratique pédagogique et ne jamais perdre de vue leur but commun, qui est le perfectionnement de l'enfant» (4). Cette discipline doit user d'émotion et de persuasion, afin de toucher les sentiments de l'élève; elle doit atteindre également l'intelligence et la raison par des démonstrations; enfin, elle doit parvenir à motiver la volonté, en suscitant des résolutions et en provoquant des actes. De ces différents éléments dépend la qualité de

(1) R.A., Doin, 1897.

(2) R.I., Laclef, 1906 et 1910.

(3) R.I.A., Seine, 1903.

(4) R.I.A., Seine, 1912. 
la formation morale et le travail des inspecteurs consiste à faire comprendre leurs articulations, par de nombreux exemples pratiques et des recommandations générales renouvelées.

Le premier élément que l'inspection se voit dans l'obligation de rappeler aux maîtres est l'attention qu'ils doivent porter à leur public. L'enseignant doit s'adapter «aux situations diverses des enfants» (1): il doit, à la fois, veiller à «choisir des sujets convenant au milieu» (2), tenir compte «de la spécificité du pays», des «circonstances locales» (3), comme de l'âge ou du sexe de ses élèves. Sur ce dernier point, une inspectrice de la Seine rappelle ainsi, en 1906, que les jeunes filles ne doivent pas recevoir un enseignement identique à celui des garçons, en particulier dans les «arrondissements du Paris ouvrier», où l'on doit être attentif à développer le «sentiment de la pudeur». De ce premier rappel découlent les critiques fréquentes des inspecteurs à l'encontre des leçons trop théoriques, voire dogmatiques, et qui dépassent les capacités de compréhension des élèves. $\mathrm{Si}$ «la leçon porte trop haut, passe par-dessus la tête de l'enfant, le laisse distrait, indifférent ou même rebuté par l'effort qu'il a fallu faire pour saisir la pensée du maître» (4), si elle se contente de «on doit, il faut, cela porte peu» (5). Ces recommandations s'inscrivent avant tout contre les procédés de la méthode synthétique ou déductive et l'usage abusif des livres scolaires. Critiques envers l'abus du manuel dans les classes, ceux que les inspecteurs produisent, dans la décennie 1900, sont de fait assez différents des ouvrages jusqu'alors publiés. Les instituteurs ne s'y trompent pas, qui inscrivent majoritairement ces manuels de morale sur les listes qu'ils dressent après 1905, alors qu'ils continuent de préférer les ouvrages des pédagogues de renom, pour l'histoire. Accordant à la leçon orale du maître une importance primordiale, les inspecteurs, auteurs d'ouvrages scolaires, rappellent que le manuel «serait inutile, et même nuisible, si l'instituteur pouvait croire qu'il suffit de faire apprendre nos résumés sans exposer ses leçons » (6), 1906. L'inspection n'incite pas les maîtres à se passer du livre scolaire puisqu'elle sait que celui-ci constitue, pour les enseignants débutants ou peu sûrs,

(1) R.I., Bony, 1908.

(2) R.I., Bacqué, 1905.

(3) R.I., Fautras, 1893.

(4) R.I.A., Seine, 1901.

(5) R.I., Bony, 1909.

(6) Delage et Charton: Morale et instruction civique, 1880. 
un «précieux auxiliaire» qui leur fournit «un choix tout fait de bons exemples» (1). De même, elle reconnaît que le manuel représente, pour les plus grands des élèves, un «guide» qui coordonne «les principales vérités» et fournit «un recueil méthodique des principales idées qu'ils devront avoir présentes à la mémoire» (2). Les inspecteurs qui rédigent des manuels témoignent donc d'un souci de renouveler l'usage du livre scolaire. Les ouvrages des inspecteurs Boyer (1895) et Bancal (1897), destinés aux maîtres, ne proposent ainsi que des supports à la préparation des leçons (3). Dans les manuels destinés aux élèves, les inspecteurs insistent d'ailleurs sur le souci qu'ils ont eu «en les écrivant, de penser au petit peuple des écoles [...] que nous connaissons très bien » (4). Leur démarche se veut une réponse «aux tâtonnements qui ont été constatés par tous ceux qui à un degré quelconque participent à la direction des études» (5). Point d'orgue de la leçon du maître, le manuel doit venir confirmer la parole de l'enseignant. Loin du «mode d'emploi» d'un Laloi qui incite le maître à une utilisation intensive de son ouvrage (6), les inspecteurs élaborent des manuels à l'organisation structurelle stricte mais qui laisse toujours à l'enseignant le soin de construire sa leçon (7). Les ouvrages de morale des inspecteurs, postérieurs à 1895, comportent généralement des règles, des préceptes et des maximes destinés à la mémoire. Ils proposent également des «histoires morales», accompagnées «d'exemples variés [...] présentant surtout une plus grande apparence de vérité et plus d'actualité». Ils font une large place à la

(1) 71 AJ 19 - Rapport du 30-03-1889 sur l'enseignement de la morale.

(2) Conférence pédagogique de Lormes, 1885, Cahier nivernais d'histoire de l'éducation, $\mathrm{n}^{\circ} 4$, p. 26.

(3) Ces manuels du maître ne comportent que des plans, résumés, «problèmes moraux et exercices de rédaction », textes de lecture et de récitation, répartitions mensuelles et bibliographie complémentaire.

(4) Launey: Leçons de morale, 1911.

(5) Bancal: Nouveau carnet de morale, 1897.

(6) Laloi écrit que «le maître fera d'abord lire le texte, subdivision par subdivision, plusieurs fois, comme s'il s'agissait d'une leçon de grammaire». Les devoirs proposés consistent «à copier des articles du supplément où l'élève trouvera des notions nouvelles» et sur lesquelles «le maître questionnera les élèves [...] après qu'ils les auront copiés», Instruction morale et civique, 1891.

(7) Les inspecteurs Poignet et Bernat: Le livre unique de morale et d'instruction civique, 1910, offrent un manuel à la présentation claire et méthodique: «à gauche, des lectures en nombre variable et convenant toutes aux trois cours; à droite, la leçon proprement dite ou résumé des principes en trois paragraphes». L'ouvrage de Jarach, Lavabre et Batillat: Leçons de morale et d'instruction civique, 1896, se compose, sur sa page de gauche, d'une ou deux maximes, puis de paragraphes qui énoncent la leçon et enfin d'un résumé dont chaque phrase correspond aux paragraphes de l'exposé. 
vie quotidienne et aux «hommes de bien» contemporains. Les résumés, «aussi simples et clairs que possible» (1) n'ont que l'avantage de «remplacer [...] la série forcément incomplète des résumés copiés machinalement par l'élève, mal transcrits, mal disposés, mal orthographiés, et nécessairement éparpillés dans tous les cahiers de l'année» (2). «Toujours très succinct (3), [...] chaque résumé ne sera appris que lorsque le maitre l'aura développé, éclairé, vivifié, en plaçant sous les formules générales des faits bien observés par l'élève» (4). Afin de ne pas ouvrir la voie à un enseignement trop utilitaire ou trop puéril, les questions ne doivent pas être de celles «auxquelles l'élève peut répondre par un oui ou par un non, ou même par une simple phrase». Pour viser au développement constant et progressif de l'intelligence enfantine, les inspecteurs leur préfèrent des petits problèmes moraux qui «obligeront l'élève à penser» (5). Adapter les manuels aux capacités de ceux pour lesquels ils sont écrits constitue donc une application pratique de leurs recommandations quotidiennes. Sans tendre à cette simplification excessive qu'ils condamnent, plusieurs inspecteurs font un réel effort pour mettre le vocabulaire à la portée des élèves (6).

Pour lutter contre des leçons trop arides et abstraites, il faut également préférer au manuel d'autres supports d'enseignement dont les procédés reposent sur l'intuition sensible et la méthode heuristique. Le recours aux historiettes morales et aux faits vécus est vivement recommandé par l'inspection primaire. Avec les détails du récit magistral, on frappe l'imagination des enfants, on les intéresse; l'enseignement devient «concret» et ils «y prennent une part active» (7). Pour cela, il faut une «parole chaude et vibrante» par laquelle le maître «émeut son auditoire»; «s'il se montre touché et convaincu lui-même, il

(1) Boyer: Instruction morale et civique, 1895.

(2) Delage et Charton: Morale et instruction civique, 1906.

(3) Certains inspecteurs choisissent de concevoir les résumés sous la forme de résolutions exprimées à la première personne car «cette forme familière» leur paraît «plus compréhensible, surtout pour les jeunes enfants», Poignet et Bernat, op. cit, 1905.

(4) Boyer: Instruction morale et civique, 1895.

(5) Lemoine: Livret d'enseignement moral, 1902.

(6) L'originalité du manuel de Delage et Charton, pourtant assez austère puisque sans illustration et sans lecture, consiste dans le choix qu'il fait de proposer, en marge de son texte d'une demi-page, les termes principaux utilisés dans le développement.

(7) R.I., inspecteur de l'Aisne, 1913. 
convainc ses élèves» (1). Illustrée de petites gravures choisies avec soin (2), la leçon devient alors un support efficace de l'enseignement car «ce que l'œil de l'élève saisit, son esprit l'interprète, l'agrandit, le vivifie, le féconde à son profit» (3).

Les inspecteurs mettent cependant en garde les instituteurs contre une croyance excessive dans les seules vertus de l'imagination et de la sensibilité (4). À partir de 1900, ils leur préfèrent l'utilisation des faits vécus, alternative méthodologique pour développer la «morale en action». Constitués «d'exemples empruntés à la vie de l'enfant» (5), «avec le tact nécessaire» quand il s'agit «de la vie sociale et familiale» (6), ce procédé nécessite des enseignants «des leçons composées par eux-mêmes » (7). Il requiert du maître une aptitude à utiliser, sur le vif, les événements de la classe qui font naître des discussions «plutôt que des argumentations» (8). Cet enseignement, plus pratique, s'appuie sur des procédés analytiques qui doivent davantage solliciter l'intérêt de l'élève. Il leur fournit, en effet, des éléments d'analyse de leurs actes quotidiens et permet au maître d'ériger «une règle de conduite, dont il envisage les diverses faces, à l'aide d'exemples» (9). De là naît un corpus de règles, exécutables de manière immédiate dans la microsociété qu'est la classe, et où les sanctions relèvent de «l'application intelligente de la discipline scolaire comme moyen d'éducation» (10). Juge et incarnation de la loi

(1) R.I., Nézondet, 1892.

(2) Dans cette discipline, qui n'offre pas de scènes aussi clairement identifiables que celles de l'histoire nationale, il demeure ardu de caractériser les idées morales. Certains inspecteurs auteurs privilégient dès lors la reproduction d'œuvres d'art (Nonus : Résumés de leçons de morale et d'instruction civique à l'école primaire, 1904).

(3) L. Jarach: La morale par l'image, 1911.

(4) Les «enfants sont intéressés cependant, malgré le rôle passif qu'ils jouent; mais leur imagination est évidemment plus frappée par le ton, les gestes, le bon dessin fait préalablement au tableau noir - que leur cœur n'est touché et à mon avis, le but de cette leçon n'est pas atteint» (R.I., CAP, 1911).

(5) R.I., Merlet, 1899.

(6) R.I., Leloutre, 1912.

(7) R.I., Doin, 1897.

(8) R.I., Lottin, 1911.

(9) R.I., Messac, 1913.

(10) J. Trabuc, inspecteur primaire: Guide administratif et pédagogique pour les fonctions d'instituteur, 1887, p. 81. 
morale scolaire, le maître devient alors la «conscience vivante de ses élèves» (1), «leur conseil et leur refuge» (2).

Développer le jugement des enfants en s'appuyant sur leur intuition morale, conduit certains instituteurs à faire des élèves les juges de leur propre conduite. Certains enseignants proposent ainsi aux élèves des auto-évaluations sur «leurs progrès de la semaine ou de la quinzaine» (3); d'autres les engagent à des «résolutions prises à la première personne du singulier» (4) et qui établissent une sorte de contrat moral entre le maître et chaque élève. Quelques instituteurs poussent la pratique jusqu'à faire juger les actes commis par des pairs, voire par des personnages historiques ou de fiction. L'inspection reste circonspecte sur la portée de tels procédés qui suscitent plus souvent des mots que des actes. Elle met en garde les enseignants sur la valeur et l'impartialité des verdicts émis par l'enfant. Recourir à ce procédé, c'est prendre le risque, en outre, de développer, chez l'élève, un manichéisme et une intransigeance peu compatibles avec les plus hautes vertus morales. Cette méthode ne vaut donc que par l'honnêteté et la franchise des propos magistraux: le maitre doit toujours «avertir l'enfant des circonstances probables où il devra rester maître de soi» (5) et lui faire sentir la difficulté du devoir moral, la complexité de sa pratique.

Les inspecteurs préfèrent développer des méthodes où l'élève est l'acteur de l'enseignement qu'il reçoit car il faut guider sa pensée pour ramener toutes les obligations particulières à des principes généraux. User de cette méthode conduit, pratiquement, «à faire trouver par les élèves eux-mêmes les vérités morales, les règles de conduite qu'on veut leur enseigner» (6). Exercice conjoint de la conscience et de l'intelligence, cette démarche permet aux enfants de comprendre qu'ils sont libres de faire le bien ou le mal, c'est-à-dire qu'ils sont responsables de leurs actes. La résolution de petits problèmes moraux, qui «affinent» et «affirment les consciences» (7) devient, à partir de

(1) R.I., L. Merlet, 1896.

(2) On comprend dès lors les attentes de la hiérarchie à l'égard de l'instituteur qui doit offrir une image morale inaltérable car l'ensemble de ses actes relève d'un procédé éducatif.

(3) R.I., Nézondet, 1896.

(4) R.I.A., Seine, 1901.

(5) R.I.A., Seine, 1912.

(6) R.I., Fautras, 1912.

(7) R.I., Lottin, 1909. 
1900, le symbole de cette préoccupation pédagogique. Soumis la veille aux plus grands des élèves, ces problèmes moraux doivent provoquer une discussion entre les élèves et le maître et se closent par une correction magistrale. Ils engagent les enfants à discuter un point de morale pratique qu'ils «sont invités à [...] méditer en dehors des heures de classe pour chercher la solution de façon à pouvoir apporter leur réponse orale au jour fixé » (1).

Éducation de chaque instant, la morale doit être enfin le sujet de toutes les matières qui concourent à son enrichissement: dictées, récitations, chants, modèles d'écriture, problèmes de mathématiques, sujets d'histoire et lectures constituent, pour tous les pédagogues, les axes complémentaires des leçons de morale. Cette discipline crée alors «une atmosphère vivifiante d'honnêteté, d'activité et de bonté agissant comme l'air, sans que l'élève s'en doute» (2) et fait de l'école un lieu «où la morale $[\ldots]$ se respire encore plus qu'elle ne s'enseigne» (3). De ces conseils naissent des initiatives étonnantes, parfois vaines et excessives, mais qui témoignent de la recherche incessante des instituteurs pour répondre aux exigences de leur hiérarchie. Dans le département de la Seine, on crée ainsi des «tribunaux d'arbitrage», des «sociétés de bon exemple» pour lutter contre les mots déplacés et les actes violents, et «dont chaque membre s'engage à proscrire de sa conversation et de ses manières tout ce qui pourrait blesser la bienséance». Ailleurs, on instaure une sorte de pratique laïque du confessionnal: «l'instituteur appelle chaque soir, à tour de rôle, un élève qui doit apprécier sa propre conduite et dire en confidence ce qui ne l'a point satisfait lui-même» (4). Des enseignants proposent la rédaction de «notices individuelles», de «graphiques», élaborés pour informer les parents de l'évolution morale de leur enfant. Ils rédigent des «livres d'or scolaires» qui établissent «une comptabilité active des bonnes actions » des élèves afin de développer «l'émulation» morale (5).

À ces procédés des années 1900, l'inspection ajoute «l'observation individuelle des caractères» (6), c'est-à-dire prône le développement

(1) R.I.A., Seine, 1902.

(2) R.I., L. Merlet, 1896

(3) R.I.A., Seine-et-Oise, 1896.

(4) R.I.A., Seine, 1902.

(5) R.I.A., Seine, 1902.

(6) J. Trabuc: Guide administratif..., op. cit; 1887, p. 81. 
de méthodes fondées sur la psychologie de l'enfant. Cette étude des caractères donne lieu, dans la Seine, à la rédaction de «dossiers personnels», où le maître consigne «des remarques psychologiques appuyées de commentaires et d'exemples». Dans ces fiches, dont les modèles sont parfois rédigés par les inspecteurs, l'instituteur «est tenu d'inscrire ses observations sur le caractère de l'enfant, sa tendance d'esprit, sa conduite, son travail, ses progrès comme ses défaillances passagères». Le maître note également «le point précis sur lequel, au besoin, il peut agir efficacement et tenter même une greffe morale» (1). Ce procédé, qui doit toujours «respecter la personne de l'enfant» et favoriser le libre et confiant déploiement de sa personnalité, a comme «première règle de conduite» de «ne jamais imposer une tâche à un enfant dans l'unique but de le faire rester tranquille» (2). Obéissance raisonnée, fondée sur la reconnaissance et le respect du maître, et non sur la crainte, ce procédé développe un esprit de tolérance conforme aux exigences supérieures de la morale. C'est là une œuvre de psychologie fiable et immédiatement accessible, mais qui, selon l'inspection, nécessite de modifier en profondeur les conceptions qu'ont encore bien trop souvent les instituteurs de leur rôle et de celui de leurs élèves.

\section{LES RÉSULTATS DE L'ENSEIGNEMENT MORAL}

Maîtres comme inspecteurs savent bien que c'est à l'aune des pratiques et des comportements que l'on jugera de la réussite de l'enseignement moral. Si l'inspection ne détient pas l'exclusivité de cette préoccupation professionnelle, l'évolution de sa fonction l'invite à s'en soucier davantage puisqu'elle a pour mission d'évaluer les maîtres et les résultats de leurs enseignements. Sommée de dresser des bilans de l'état de cette discipline, l'inspection cherche les moyens de l'évaluer et de l'améliorer.

Optimistes, au départ, quant à la réussite de ce nouvel enseignement, les inspecteurs vont se montrer, avec le temps, toujours plus exigeants, se jugeant, ainsi que les enseignants, responsables des insuffisances constatées. Loin de magnifier les résultats, le discours des inspecteurs est au contraire trop systématiquement critique, comme le souligne l'inspecteur d'académie de la Seine en 1912. Ce

(1) R.I.A., Seine, 1901.

(2) Id., p. 85. 
dernier rappelle aux inspecteurs, qu'à l'image de l'ensemble de la formation donnée dans l'école, l'enseignement moral ne peut, au mieux, être conçu que comme une préparation de l'enfant à sa future vie de citoyen et d'honnête homme et non comme une éducation achevée et évaluable en tant que telle. Cet enseignement n'est que la première phase d'un long processus de développement individuel. Rien ne dit qu'il ne portera pas ses fruits en temps utile, perspective optimiste dont Marc Sangnier se fait, en 1917, l'écho: «Je les sentais capables de pénétrer les besoins profonds de la France, - dont ils portaient en eux les aspirations les meilleures -, et de l'humanité, que doit servir la France» (1).

Dresser le bilan de cet enseignement, entre 1880 et 1914, c'est adopter les deux critères d'évaluation sur lesquels s'appuie l'inspection. Le premier élément est le travail des maîtres qui, au-delà des insatisfactions chroniques des inspecteurs, semble en voie de constante amélioration, du moins en Seine-et-Oise. Dans la décennie 1880, les avancées les plus notables se rencontrent dans les écoles publiques de garçons, «les écoles de filles [restant] dans un état d'infériorité manifeste sous ce rapport» (2) car la majorité des enseignantes, non encore formée à cette discipline nouvelle, préfère se cantonner à celle qu'elle pratiquait antérieurement (3). Les institutrices tardent, en effet, à mettre efficacement en application les conseils donnés en conférences pédagogiques et lors des visites d'inspection. C'est pourquoi il faut attendre, selon l'inspectrice Louise Merlet, les années 1890 pour observer un réel progrès moral chez les jeunes filles des écoles laïques et les jeunes enfants des écoles maternelles.

Le renouveau voulu par les programmes des années 1880 n'apparaît pas clairement pour les enseignants formés dans les écoles normales du Second Empire. Si les conférences pédagogiques de Seine-et-Oise se constituent efficacement dès 1879 , il faut d'ailleurs attendre la décennie 1890 pour que l'on y aborde le sujet de la morale scolaire. En cela, l'inspection ne fait que confirmer les incertitudes de tous les pédagogues quant à cette nouvelle matière. Jusqu'en 1894, la progression du nombre de manuels d'instruction morale et civique

(1) M. Sangnier: Ce que savent les jeunes Français aujourd'hui. Simple contribution à une enquête sur l'instruction, Librairie «La Démocratie», 1917, 19 p.

(2) R.I.A., Seine-et-Oise, 1885-1886.

(3) Les écoles congréganistes n'enseignent qu'un programme amputé de ce qui en fait sa nouveauté, soit la morale laïque et républicaine. 
reste faible (1). Au regard de l'expansion prise par les livres scolaires à la même époque (notamment ceux d'histoire), on mesure la difficulté à proposer des ouvrages qui se distinguent de l'ancienne éducation morale et religieuse.

Les résultats atteints en 1895, en 1900, puis en 1914 ne sont jamais considérés par les inspecteurs que comme des avancées, régulières, mais toujours insuffisantes. Les progrès observés semblent tenir à l'apparition d'un élément-clé: l'existence quotidienne d'une leçon de morale à part entière, mais que les maîtres doivent encore améliorer dans son déroulement. En outre, l'enseignement de la morale n'est plus véritablement contesté: il fait désormais l'objet de nombreuses publications et il est largement traité en conférences pédagogiques. Légitimes, les exigences des inspecteurs ne nuisent d'ailleurs pas à leur compréhension des difficultés de terrain puisqu'ils continuent à prendre en considération, dans les inégalités de réussite, la diversité des structures d'écoles et la variété des publics scolaires.

Par ailleurs, l'évaluation des résultats de cet enseignement est commune à tous ceux qui se préoccupent de la formation morale des plus jeunes: on juge de ses progrès soit à l'aune de l'amélioration des comportements enfantins, soit au regard d'une élévation de la moralité des élèves (2). Cette systématisation à rapporter les attitudes enfantines, individuelles ou collectives, se traduit par une observation du comportement social des élèves. En 1901, les enfants sont ainsi estimés «plus doux dans leurs gestes, plus modérés dans leurs propos» selon l'inspecteur d'académie de la Seine. La franchise, la bonne camaraderie et l'esprit de solidarité prennent le pas sur les anciens défauts. Les élèves témoignent de plus de probité et, alors que «la misère augmente», on observe moins de larcins, affirme la directrice «d'une école d'un quartier pauvre». Ces analyses, militantes quand elles déprécient l'attitude des enfants des écoles prérépublicaines, fournissent également aux partisans du nouvel enseignement moral des indices concrets permettant de souligner sa valeur auprès des populations influencées par les détracteurs de l'école laïque. Au-delà de l'adéquation aux normes morales établies,

(1) L'étude des listes départementales établissant les manuels autorisés et celle des registres du dépôt légal corroborent ce constat.

(2) Le ministre préconise lui-même cette méthode, dès 1883, dans sa Lettre aux instituteurs. 
les actes charitables quotidiens, la «morale en action», deviennent donc des signes tangibles de ce qui apparaît comme une amélioration morale des enfants.

À cette évaluation des comportements quotidiens des élèves s'ajoute, pour les inspecteurs, celle de leur aptitude à porter un jugement sur un personnage, un événement, historique ou imaginaire. L'usage de ce procédé n'est pas secondaire et correspond à une démarche dont la finalité est plus proche des idéaux supérieurs voulus par les législateurs dans l'éducation morale. Il s'agit alors de former une conscience libre, éclairée, raisonnée et fondée sur ces principes de la dignité humaine et de la solidarité sociale, leitmotive qui concluent presque tous les rapports de l'inspecteur de Seine-et-Oise, C. Lottin.

Cette observation des comportements enfantins s'étend, dans la décennie 1890, à celle des jeunes adultes sortis des classes primaires de la République. En effet, l'amenuisement des contestations de la morale laïque favorise ces analyses. De plus, le débat qui naît, après 1893, sur la responsabilité de l'école laïque dans la montée de la délinquance juvénile, comme de la criminalité en général, le nécessite. Honnête et réaliste, l'inspection primaire reconnaît d'ailleurs que ces attitudes, en voie d'amélioration, ne constituent pas encore des habitudes et que les «impressions que reçoivent les enfants s'effacent vite à la sortie de l'école» (1). À ces critiques, l'inspecteur primaire de Versailles rappelle que «ce n'est pas en douze ans qu'on peut opérer des changements très sensibles »; «les jeunes gens qui ont reçu l'enseignement moral en 1883 sortent à peine du régiment. Il faut attendre qu'ils soient leurs maîtres, qu'ils aient une famille à élever, pour savoir au juste ce qu'ils sont et ce qu'ils valent». Pour répondre aux accusations des détracteurs de «l'école sans Dieu», l'inspecteur Bacqué évoque, dès 1896, d'autres facteurs: "Dans les pays où l'enseignement n'est pas laïque, l'augmentation [de la criminalité] se manifeste autant que chez nous, sinon plus. Elle tient aux conditions mêmes de l'existence actuelle, à une transformation de la vie sociale, à une soif de luxe et de jouissances matérielles et à diverses causes indépendantes de l'école».

Selon l'inspection, la réussite de l'enseignement de la morale tient à deux éléments qui peuvent facilement devenir des obstacles de

(1) R.I.A., Seine-et-Oise, 1912. 
taille: la famille et les maîtres. «Indispensable» au succès de l'éducation morale, «le concours de la famille» est, selon l'inspecteur Martin, encore rare en 1893. À ce désintérêt des parents, voire à leur mauvais exemple s'ajoutent les dangers extérieurs rencontrés par l'enfant. Pour l'inspecteur Plâtrier, chaque milieu social recèle ses écueils: la ville où «les occasions sont fréquentes", où «l'entraînement est grand» et où l'enfant «se trouve alors exposé à tous les dangers de la rue, du cabaret, des journaux et des livres malsains »; les centres industriels où «le mal est pire que partout ailleurs»; enfin, les campagnes, où la dépravation subsiste bien qu'elle soit «plus restreinte et moins efficace» (1). À ces causes largement reprises par l'ensemble de la presse pédagogique dès 1900, s'ajoutent celles qu'évoque l'inspecteur d'académie de la Seine, en 1912: «Désordre de notre civilisation industrielle, manque d'enseignement professionnel, crise de la puberté, désorganisation de la famille, voire désarroi des croyances et des idées sont des causes qui produisent dans tous les pays d'Europe les mêmes effets ».

L'inspection ne peut se contenter de dénoncer cette situation. Son rôle l'oblige à trouver les moyens d'établir, entre l'école et les familles, une interaction éducative, «l'autorité morale des maîtres n'ayant rien à gagner à ce divorce entre les deux principales forces d'éducation du temps présent» (2). Ces réflexions jettent les bases d'une nouvelle relation entre maîtres et parents, significatives de la volonté des pédagogues républicains d'imposer, par l'enseignement moral des plus jeunes, les nouveaux fondements sociaux et civiques de la nation.

C'est donc au maître que l'on confie le rôle de convaincre, avec tact et persuasion, les familles de l'importance de leur participation à l'œuvre éducative. Cette mission est d'autant plus difficile à mener que l'incompétence des enseignants constitue, pour les inspecteurs, la seconde cause des insuffisances de l'enseignement moral. Si l'éducation première, la formation reçue ensuite (3), puis les pratiques péda-

(1) R.A., Plâtrier, 1893.

(2) R.I.A., inspecteur du $\mathrm{XV}^{\mathrm{e}}$ arrondissement de la Seine, 1901.

(3) Dans les années 1880, l'inspection met en cause le manque de formation de maîtres recrutés sous le Second Empire et non formés à l'enseignement moral républicain. Cependant, elle renouvelle ce constat en 1910, les besoins accrus du service ayant conduit à recruter des maîtres d'une instruction "peu étendue, d'un savoir pédagogique plutôt faible». 
gogiques des instituteurs sont particulièrement critiquées par la hiérarchie, c'est que cette matière exige d'eux «un effort plus personnel, une action plus continue, une attention plus en éveil» (1). Remédier à ces défauts professionnels est l'objet des conseils donnés dans le cadre des inspections ou des conférences pédagogiques. Face à cette situation, les inspecteurs proposent des solutions pratiques immédiates, tel que l'abandon du manuel au bénéfice de la préparation écrite personnelle des leçons par les instituteurs et la tenue de carnets de morale par les élèves. Leurs conseils sont suivis d'effets : les «carnets de notes et de résumés spéciaux de morale» sont en usage, à partir de 1895-1900, dans un grand nombre d'écoles de la Seine-et-Oise et de la Seine et l'inspection observe une plus grande diversification des procédés d'enseignement. Aux récits et entretiens familiers dont ressortent des «conseils à la portée» des plus jeunes, l'inspectrice Merlet relève, en 1896, l'ajout de lectures expliquées, de maximes développées ou d'exposés oraux, accompagnés d'interrogations et de résumés, pour les plus grands. Vingt ans après les lois scolaires, les enseignants témoignent d'un plus grand désir de s'instruire, de «voir clair dans leur pensée comme dans l'âme de leurs élèves » (2). Ils ont compris «la haute importance du but», même si certains se trouvent toujours en proie à «l'hostilité qui, en ces dernières années, a recommencé contre l'enseignement d'une morale indépendante» ou aux attaques des plus zélés défenseurs de la laïcité qui reprochent encore à quelques institutrices des «tendances à continuer indirectement l'enseignement religieux ». Jugée notable, l'évolution des résultats et des méthodes d'enseignement reste cependant insuffisante et de nombreux écueils menacent toujours les enseignants quels que soient les procédés qu'ils emploient.

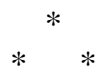

En nous attachant aux pratiques de classe, nous avons tenté de présenter ce que recouvre l'enseignement de la morale dans l'école de la Troisième République. Vivement discuté, à la Chambre comme dans l'opinion publique, pour ses enjeux éducatifs qui rompaient avec son ancien statut, cet enseignement le fut aussi par ceux qui, au quotidien, avaient pour mission de l'enseigner. Acteurs des ambitions formatives du gouvernement républicain, les inspecteurs primaires

(1) R.I.A., Seine, 1901

(2) R.I.A., Seine, 1902. 
témoignent de leur engagement, comme de celui des maîtres dont ils rapportent la diversité des initiatives, pour faire triompher cette nouvelle discipline. Parallèlement, on constate que les finalités de cette morale scolaire modifient le rôle assigné à ces fonctionnaires et l'exercice même de leur métier. Cette matière doit en effet être envisagée autrement que comme une simple instruction: elle ne doit pas seulement «faire savoir», elle doit «faire pratiquer». À la différence d'autres disciplines, elle nécessite dès lors de l'inspection une vision qui aille au-delà des seuls contenus d'enseignement. Les objectifs supérieurs de l'éducation morale conduisent par conséquent ceux qui sont chargés de son succès à s'interroger, d'une part sur la façon d'enseigner, d'autre part sur l'élaboration de critères permettant d'évaluer la réussite de cet enseignement. Ces questions éclairent d'un jour nouveau ce que fut la morale laïque de l'école primaire républicaine dans ses objectifs, ses contenus et ses méthodes d'enseignement. Au centre d'un nouveau débat dans la décennie 1910, ces dernières constituent un aspect essentiel du travail de l'inspection primaire, dès la fin des années 1890. L'importance de l'enseignement de la morale se mesure à l'existence de trois éléments que l'on ne retrouve pas pour d'autres disciplines. D'une part, l'apparition d'une réflexion sur les raisons de sa plus ou moins grande réussite, soit des constats d'ordre social ou professionnel. D'autre part, le développement de pratiques pédagogiques spécifiques, dans le déroulement des séquences d'enseignement et les outils à utiliser. Enfin, la réussite de ce projet d'éducation développe l'attention portée à la psychologie de l'enfant - soit la prise en compte de son âge, de son intérêt et de son activité lors de ses apprentissages.

Les réflexions et réalisations pédagogiques des acteurs de cette formation montrent qu'elle est moins normative que ne le laissent supposer les programmes officiels et les contenus des manuels scolaires. Les pratiques de classe des années 1910 soulignent combien les aspirations de l'enseignement de la morale, définies dans la décennie 1880 et désormais acceptées et comprises, restent des objectifs d'éducation supérieurs.

Delphine MERCIER Docteur en histoire 